\title{
Virtual reality exposure was as effective as standard exposure for reducing fear of flying
}

Rothbaum BO, Hodges $L$, Smith S, et al. A controlled study of virtual reality exposure therapy for the fear of flying.

J Consult Clin Psychol 2000 Dec;68:1020-6.

\section{QUESTION: In adults with fear of flying, is virtual reality exposure (VRE) as effective as standard exposure (SE) for reducing fear and avoidance behaviour?}

Design

Randomised (unclear allocation concealment*), unblinded*, controlled trial with 12 months of follow up.

Setting

Atlanta, Georgia, USA.

\section{Patients}

49 adults who had flown before and who met DSM-IV criteria for specific phobia, situational type, panic disorder with agoraphobia, or agoraphobia without a history of panic disorder with flying as the feared stimulus. Exclusion criteria were history of mania, schizophrenia, or other psychoses; prominent suicidal ideation; or current substance abuse or dependence. 45 adults (92\%, mean age $41 \mathrm{y}, 71 \%$ women) completed follow up at 6 weeks.

\section{Intervention}

Patients were allocated to 1 of 3 groups for 6 weeks: VRE $(n=15), S E(n=15)$, or a waiting list control $(n=15)$. VRE and SE consisted of 8 sessions each, 4 of which involved information gathering, treatment planning, brief breathing retraining, and cognitive restructuring. VRE was done in an office and involved simulations of sitting in an airplane, experiencing takeoffs and landings, and flying in both calm and stormy weather twice weekly for 2 weeks. $\mathrm{SE}$ was done at the airport with exposure to pre-flight stimuli and sitting on a stationary airplane with imaginal exposure to takeoffs, cruising, and landing.

National Institute of Mental Health and

Virtually Better, Inc.

For correspondence: Dr B O Rothbaum

Department of

Psychiatry and

Behavioral Sciences,

The Emory Clinic,

1365 Clifton Road,

Atlanta, GA 30322,

USA.Fax +1404778

4655 .

\section{Main outcome measures}

Proportion of patients who went on a round trip flight at 6 weeks, and changes in symptoms (Questionnaire on Attitudes Toward Flying [QAF] and the Fear of Flying Inventory $[\mathrm{FFI}])$.

\section{Main results}

More patients in the 2 treatment groups than in the control group flew at 6 weeks $(\mathrm{p}<0.01$ for both

Virtual reality exposure (VRE), standard exposure (SE), or waiting list (WL) for fear of flying $\dagger$

\begin{tabular}{|c|c|c|c|c|}
\hline $\begin{array}{l}\text { Outcomes at } \\
6 \text { weeks }\end{array}$ & Comparisons & Event rates & $\mathrm{RBI}(95 \% \mathrm{Cl})$ & NNT (CI) \\
\hline \multirow[t]{3}{*}{ Went on flight } & VRE $v$ WL & $53 \% \vee 7 \%$ & $\begin{array}{l}700 \% \\
\text { (60 to } 4569)\end{array}$ & 3 (2 to 7$)$ \\
\hline & SE $v \mathrm{WL}$ & $67 \%$ v $7 \%$ & $\begin{array}{l}900 \% \\
(108 \text { to } 5643)\end{array}$ & 2 (2 to 4$)$ \\
\hline & & $\begin{array}{l}\text { Unadjusted mean } \\
\text { difference }(95 \% \mathrm{Cl})\end{array}$ & Effect size & $\begin{array}{l}\text { Proportion of } \\
\text { controls with } \\
\text { worse outcome }\end{array}$ \\
\hline \multirow[t]{2}{*}{ QAF } & VRE $v$ WL & 70.7 (21.4 to 120.0$)$ & 0.51 & $70 \%$ \\
\hline & SE $v \mathrm{WL}$ & 59.6 (5.1 to 114.2$)$ & 0.40 & $66 \%$ \\
\hline \multirow[t]{2}{*}{ QAF fear item } & VRE $v$ WL & $3.3(1.9$ to 4.6$)$ & 0.70 & $76 \%$ \\
\hline & SE $v \mathrm{WL}$ & 2.8 (1.2 to 4.4$)$ & 0.62 & $73 \%$ \\
\hline $\mathrm{FFI}$ & VRE $v$ WL & $32.7(-5.2$ to 70.5$)$ & 0.21 & $58 \%$ \\
\hline
\end{tabular}

$\dagger \mathrm{FFI}=\mathrm{Fear}$ of Flying Inventory; QAF=Questionnaire on Attitudes Toward Flying. Other abbreviations defined in glossary; RBI, NNT, mean differences, and $\mathrm{Cl}$ calculated from data in article.

fProportion of adults in control group with worse outcome than average adult in treatment group; see glossary. comparisons) (table). The VRE and SE groups had lower (better) mean scores than the control group on the QAF and the QAF Fear item at 6 weeks; the VRE group also had lower scores than the control group on the FFI (table). At 6 months, the 2 treatment groups maintained their treatment gains and did not differ in symptom scores.

\section{Conclusion}

In adults with fear of flying, virtual reality exposure was as effective as standard exposure for reducing fear and avoidance behaviour.

*See glossary.

\section{COMMENTARY}

The study by Rothbaum $e t$ al is a step forward in the use of technology to assist in psychological treatment. The application of technology in this way is becoming more widespread, but what has been lacking is the rigorous evaluation of these new approaches. Some outcome studies have been reported. These include evaluations of standalone computer programs, computer assisted telephone and internet based interventions, and mobile computing technology. ${ }^{1}$ In the mobile computing study, the aim was to make available the tools of therapy within the patient's environment so that outcomes might be improved. In contrast, VRE therapy uses the computing technology to replicate the feared stimuli in the therapist's office. The comparison of SE with VRE allowed some conclusions to be drawn about the value of the technology compared with usual practice. The limitation on these conclusions is the lack of statistical power in the study. In addition, what is not known is any specific therapeutic effect of the technology. That is, does the use of VRE provide any additional advantage beyond convenience? Imaginal exposure is a relatively straightforward procedure; however, the effectiveness of exposure is limited by the extent to which a patient can generate the feared stimuli and, at times of distress, continue the exposure. Traditionally, this task is the domain of the therapist. If VRE can facilitate this by creating a greater sense of control by the patient over the exposure experience, and possibly accelerate recovery, then it has the potential to supersede traditional imaginal exposure.

Although VRE is a technology that has been available for some time, the cost and ease of use have been greatly improved recently, and is thus within the reach of many mental health practitioners. The findings are promising for the future of exposure but at this stage it can only be concluded that VRE provides an effective medium for exposure, rather than a treatment itself.

Justin Kenardy, $\mathrm{PhD}$ University of Queensland, Brisbane, Queensland, Australia

1 Newman MG, Kenardy J, Herman S, et al. Comparison of palmtop-computer-assisted brief cognitive-behavioral treatment to cognitive-behavioral treatment for panic disorder. $J$ Consult Clin Psychol 1997;65:178-83. 